02

\title{
Квантовая динамика заряда в квазиодномерных сверхпроводниках
}

\author{
(C) Я.С. Лехтинен ${ }^{1}$, Б.Г. ЛЬвов ${ }^{2}$, К.Ю. Арутюнов ${ }^{2,3, \text { ฯ }}$ \\ ${ }^{1}$ VTT Technical Research Centre of Finland Ltd, \\ Espoo, Finland \\ ${ }^{2}$ Национальный исследовательский университет „Высшая школа экономики“, \\ Московский институт электроники и математики, \\ Москва, Россия \\ ${ }^{3}$ Институт фризических проблем РАН им. П.Л. Капицы, \\ Москва, Россия \\ ๑ E-mail: karutyunov@hse.ru
}

\begin{abstract}
Экспериментально исследовались вольт-амперные характеристики тонких сверхпроводящих нанопроводов из титана. Наиболее тонкие образцы, измеряемые высокомными контактами, обнаружили нетривиальное для сверхпроводника поведение: кулоновскую блокаду. Величина кулоновской щели коррелирует с частотой квантовых проскальзываний фазы. Наблюдение подтверждает идентичность квантовой динамики заряда в джозефсоновских контактах и в квазиодномерных сверхпроводящих каналах в режиме квантовых флуктуаций параметра порядка.
\end{abstract}

В работе использованы материалы проекта ТЗ-93 „Квантовые твердотельные системы“ в рамках Программы „Научный фонд Национального исследовательского университета “ Высшая школа экономики (НИУ ВШЭ)“ с использованием средств субсидии на государственную поддержку ведущих университетов Российской Федерации в целях повышения их конкурентноспособности среди ведущих мировых научнообразовательных центров, выделенной НИУ ВШЭ.

DOI: 10.21883/FTT.2018.11.46645.04NN

\section{1. Введение}

Яркое проявление флуктуационных эффектов является специфической особенностью низкоразмерных систем по сравнению с массивными объектами. В квазиодномерных сверхпроводящих каналах при рассмотрении флуктуаций сверхпроводящего параметра порядка $\Delta=|\Delta| e^{i \varphi}$, являющегося комплексной величиной, необходимо различать флуктуации фазы $\varphi$ и модуля $|\Delta|[1]$. Было обнаружено, что квантовые флуктуации модуля $|\Delta|$ приводят к размытию щелевой особенности [2,3], а топологические сингулярности фазы - квантовые проскальзывания фазы - влияют как на транспортные [4], так и - термодинамические свойства - незатухающие сверхпроводящие токи $[5,6]$.

Существует фундаментальная связь между процессами, происходящими в джозефсоновских системах и в тонких сверхпроводящих каналах в режиме квантовых флуктуаций [7]. Можно утверждать, что квантовое проскальзывание фазы - динамический эквивалент статического (в пространстве и времени) джозефсоновского контакта. Продолжая качественную аналогию, правомочно заметить, что транспортные свойства достаточно тонкого сверхпроводника в низкоомном окружении [8] эквивалентны свойствам смещенной по напряжению $V$ джозефсоновской системы. В этом случае фаза $\varphi$ сверхпроводящего параметра порядка является квазиклассической величиной, и ее временная эволюция описывается хорошо известным соотношением: $d \varphi / d t=2 e V / h$. Ситуация качественно меняется, если система смещена по току [9]. Тогда, вместо фазы $\varphi$, заряд $q$ дол- жен рассматриваться как квазиклассическая переменная. В этом режиме квантовая динамика заряда формально описывается выражениями, идентичными движению блоховского электрона в периодическом потенциале кристаллической решетки $[10,11]$. Целью работы является экспериментальное исследование этого явления.

\section{2. Теория}

В теоретических работах $[7,12]$ было показано, что физические свойства сверхпроводящего провода в режиме квантовых флуктуаций описываются гамильтонианом

$$
\hat{H}_{Q P C}=\frac{E_{L}}{(2 \pi)^{2}} \hat{\varphi}^{2}-E_{Q P C} \cos (2 \pi \hat{q})+\hat{H}_{c o u p}+\hat{H}_{e n v},
$$

который дуален гамильтониану джозефсоновского контакта

$$
\hat{H}_{J}=E_{C} \hat{q}^{2}-E_{J} \cos \hat{\varphi}+\hat{H}_{\text {coup }}+\hat{H}_{\text {env }}
$$

при замене канонически сопряженных операторов заряда $\hat{q}$ и фазы $\hat{\varphi}$, связанных коммутационным соотношением $[\hat{q}, \hat{\varphi}]=-i$. Член $\hat{H}_{e n v}$ описывает электромагнитное окружение, а $\hat{H}_{\text {coup }}$ отвечает за взаимодействие системы с этим окружением. Величины $E_{Q P S}, E_{C}, E_{L}$ и $E_{J}$ - характерные энергии, ассоциируемые с частотой квантовых флуктуаций, емкостью, индуктивностью и джозефсоновской связью, соответственно. Простое сравнение двух гамильтонианов (1) и (2) показывает их идентичность при замене переменных

$$
E_{c} \leftrightarrow \pi^{2} E_{L}, E_{J} \leftrightarrow 2 E_{Q P C}, \varphi \leftrightarrow \pi q / 2 e .
$$


В конкретном случае сверхпроводящего провода, последовательно соединенного с высокоомным окружением $R_{S} \gg R_{Q}=h /(2 e)^{2}=6.47 \mathrm{k} \Omega$ и смещенного по току, при выполнении условия $E_{Q P S} \gg E_{L}$, падение напряжение описывается простым соотношением

$$
V(t)=V_{C} \sin (2 \pi q)+2 e\left(L \frac{d^{2} q}{d t^{2}}+R_{S} \frac{d q}{d t}\right) .
$$

Выражение (4) идентично хорошо исследованному в физике джозефсоновских контактов потенциалу частицы, движущейся на „наклоненной стиральной доске“, с точностью, что ток через контакт $I$ заменяется на напряжение на проводе $V$; и соответствующая координата заряд $q$, а не фаза $\varphi$. Критический ток контакта $I_{C}$ заменяется на критическое напряжение $V_{C}=(2 \pi / 2 e) E_{Q P S}$. Описанная идентичность устраняет формальное отличие двух систем с точки зрения квантовой динамики. Джозефсоновский контакт с прозрачным барьером идентичен сверхпроводнику с критическим током $I_{C}$, а сверхпроводящий провод - цепочке туннельных контактов, демонстрирующих кулоновскую щель $\delta V_{C B}=V_{C}$. Анализ выражения (4) приводит к, на первый взгляд, парадоксальному выводу: в области малых смещений по напряжению тонкий сверхпроводящий канал в режиме сильных квантовых флуктуаций должен проявлять диэлектрическое поведение: обнаруживать кулоновскую блокаду с критическим напряжением $V_{C}$. Приведенный выше краткий обзор составляет теоретический фундамент настоящего исследования.

\section{3. Образцы и методика эксперимента}

Методом взрывной электронно-лучевой литографии и направленного многоуглового вакуумного напыления были изготовлены наноструктуры на базе титановых нанопроводов с высокоомными подводящими контактами из висмута (рис. 1). Для исследования квантовых размерных эффектов использовался метод неразрушающего уменьшения размеров наноструктуры травлением в направленном пучке низкоэнергетической плазмы инертного газа [13]. Методика позволяет исследовать размерный эффект на одном и том же образце при уменьшении характерного размера с шагом до $1 \mathrm{~nm}$. При этом было показано, что при ускоряющих энергиях порядка $500 \mathrm{eV}$ глубина проникновения бомбардирующих ионов $\left(\mathrm{Ar}^{+}\right)$ в матрицу титана - не более $2 \mathrm{~nm}$, что сравнимо с толщиной естественного оксидного слоя. Метод исключительно полезен для надежной интерпретации эксперимента, когда неизбежные дефекты конкретной наноструктуры могут замаскировать тонкие размерные эффекты. Дополнительным преимуществом метода является сверхтонкая полировка поверхности, приводящая после нескольких циклов ионного травления к шероховатости поверхности на уровне $\pm 1 \mathrm{~nm}$. Геометрия образцов контролировалась сканирующим электронным и

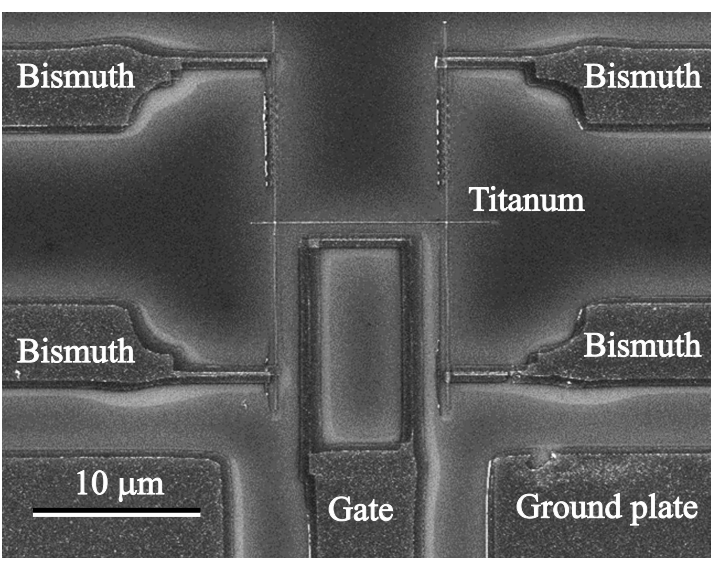

Рис. 1. Электронная микрофотография структуры.

контактно-силовым микроскопами [13]. Измерения проводились при сверхнизких температурах в рефрижераторе растворения ${ }^{3} \mathrm{He}^{4} \mathrm{He}$. Все входные/выходные линии тщательно фильтровались для уменьшения паразитного вклада внешних электромагнитных наводок.

\section{4. Результаты и обсуждение}

В четырехконтактной конфигурации исследовались $R(T)$ и $V-I$ характеристики титановых нанопроводов идентичной геометрии (рис. 1). Длина самой тонкой части („тело“ образца) была одинаковой - $10 \mu \mathrm{m}$, а эффективный диаметр (квадратный корень из сечения) варьировался от $\sim 50 \mathrm{~nm}$ до $15 \mathrm{~nm}$ размеров. В полном соответствии с более ранними наблюдениями [14], все тонкие наноструктуры продемонстрировали размытые $R(T)$ характеристики, форма которых хорошо описывается вкладом квантовых флуктуаций параметра порядка $[1,15,16]$.

Вольт-амперные характеристики (BAX) самых тонких образцов с эффективным диаметром и висмутовыми электродами с сопротивлением порядка $10 \mathrm{M} \Omega$ демонстрируют четкую кулоновскую блокаду (рис. 2). Величина кулоновской щели $\delta V_{C B}$ хорошо совпадает с оценкой $\delta V_{C B}=E_{Q P S} / e$, полученной из данных по фитированию $R(T)$ зависимостей аналогичных образцов (с низкоомными контактами) моделью $[1,15,16]$. Наблюдаемая кулоновская щель квазипериодически модулируется напряжением на „затворе“ - близкорасположенным электродом, емкостным образом связанным с образцом [17]. Период осцилляций находится в разумном согласии с соответствующей геометрической емкостью. При малых токах смещения $\leq 50$ рА экспериментальная BAX скачкообразно переключается с токонесущего в изолирующее состояние. Подобную зависимость следует ожидать в случае S-образной BAX. Соответствующий „загиб“ ВАX („блоховский нос“) (рис. 2) - прямое следствие нетривиальной электродинамики заряда (вы- 

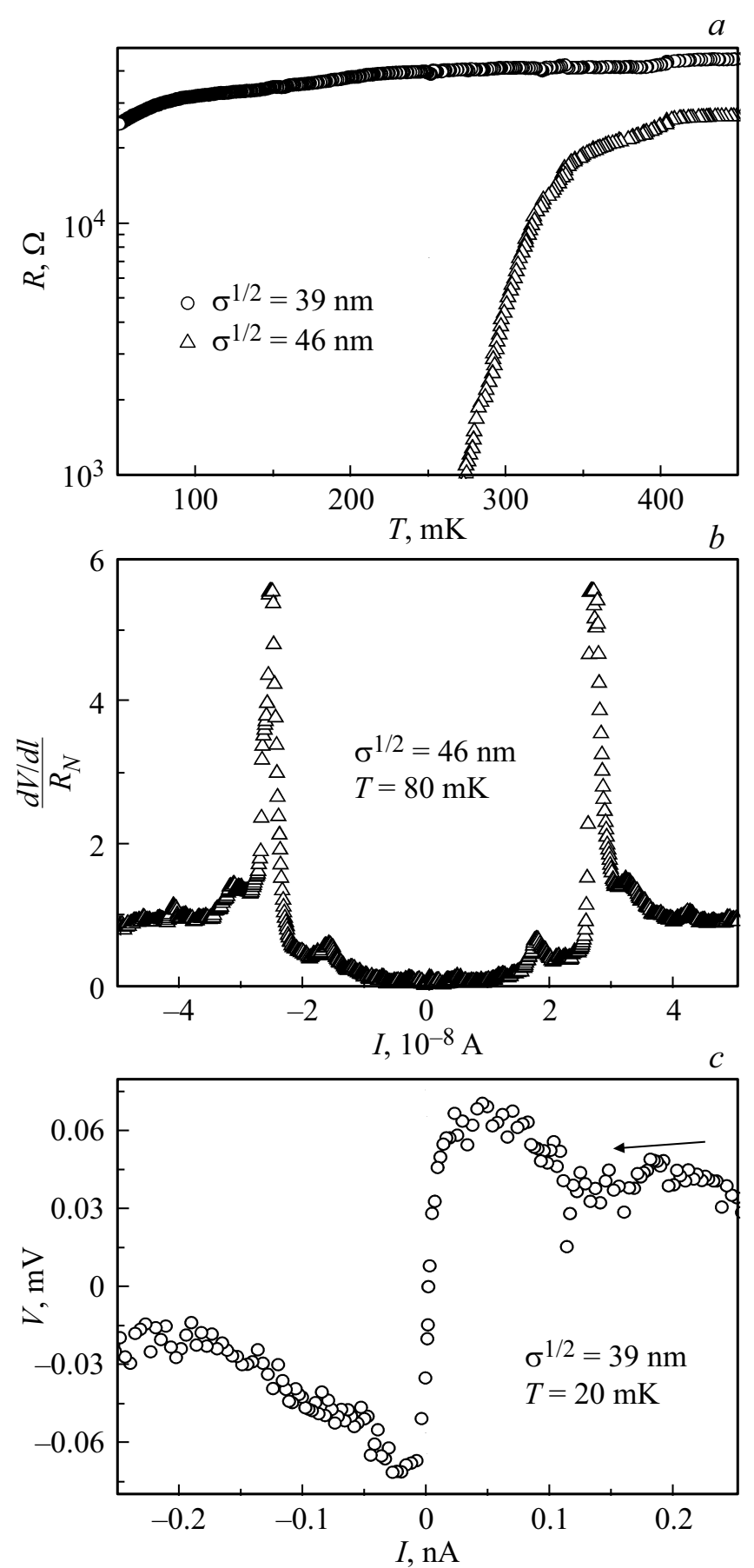

Рис. 2. $a-$ температурные зависимости сопротивления двух титановых нанопроводов с эффективным диаметром (квадратный корень из сечения $\sigma$ ) порядка $39 \mathrm{~nm}$ и $46 \mathrm{~nm}$; $b$ - дифференциальная вольт-амперная характеристика $46 \mathrm{~nm}$ нанопровода при температуре $T=80 \mathrm{mK}$, нормированная на сопротивление в нормальном состоянии $R_{N}$. Предположительно, сингулярность при токе около $\pm 25 \mathrm{nA}$ соответствует переходу в резистивное состояние всей наноструктуры, в то время как при $\pm 18 \mathrm{nA}$ - только малой части со сверхпроводимостью, ослабленной эффектом близости с электродами из нормального металла (висмута). $c$ - вольт-амперная характеристика $39 \mathrm{~nm}$ нанопровода при температуре $T=20 \mathrm{mK}$. Стрелкой обозначено направление записи данных. ражение (4)) в квазиодномерных сверхпроводниках в режиме квантовых флуктуаций параметра порядка.

Следует заметить, что нелинейные особенности на BAX исчезают при температурах выше $\sim 400 \mathrm{mK}$, что близко по величине с критической температурой $T_{C}$ титановых пленок. Предположим, что поперек нанопровода с характерным диаметром порядка $30 \mathrm{~nm}$ случайным образом сформировался один (или несколько) туннельных барьеров. Элементарный расчет показывает, что величина одноэлектронной кулоновской щели ( зарядовой энергии) эквивалентна температуре $\sim 7 \mathrm{~K}$. В этом случае, сильно нелинейные ВАХ должны были бы наблюдаться и выше критической температуры титана. Отсутствие эффекта при $T>T_{C}$ подтверждает предположение, что эффект связан со сверхпроводящими свойствами, а не с тривиальным вкладом туннелирования через статические в пространстве и времени слабые связи. Наблюдение подтверждает идентичность квантовой динамики заряда в джозефсоновских контактах $[18,19]$ и в квазиодномерных сверхпроводящих каналах в режиме квантовых флуктуаций параметра порядка.

\section{5. Заключение}

Были исследованы BAX тонких титановых нанопроводов. Наблюдаемые при сверхнизких температурах нелинейные BAX интерпретируются на базе модели, рассматривающей с единой позиции динамику заряда в джозефсоновских контактах и квазиодномерных сверхпроводящих каналах в режиме квантовых флуктуаций параметра порядка. Хочется верить, что наши эксперименты послужат стимулом для дальнейших исследований, в том числе теоретических.

\section{Список литературы}

[1] K.Yu. Arutyunov, D.S. Golubev, A.D. Zaikin. Phys. Rep. 464, 1 (2008).

[2] K.Yu. Arutyunov, J.S. Lehtinen. Nanoscale Res. Lett. 11, 364 (2016).

[3] К.Ю. Арутюнов, Я.С. Лехтинен, А.А. Радкевич, А.Г. Семенов, А.Д. Заикин. ФТТ 59, 11, 2092 (2017).

[4] K.Yu. Arutyunov, A. Ramos-Álvarez, A.V. Semenov, Yu.P. Korneeva, P.P. An, A.A. Korneev, A. Murphy, A. Bezryadin, G.N. Gol'tsman. Nanotechnology 27, 47LT021 (2016).

[5] K.Yu. Arutyunov, T.T. Hongisto, J.S. Lehtinen, L. Leino, A. Vasiliev. Sci. Rep. 2, 213 (2012).

[6] K.Yu. Arutyunov, T.T. Hongisto. Phys. Rev. B 70, 064514 (2004).

[7] J. Mooij, Y.V. Nazarov. Nature Phys. 2, 169 (2006).

[8] J.S. Lehtinen, K.Yu. Arutyunov. Supercond. Sci. Technol. 26, 039503 (2013).

[9] K.Yu. Arutyunov, J.S. Lehtinen, T. Rantala. J. Supercond. Nov. Magn. 29, 569 (2016).

[10] D.V. Averin, A.B. Zorin, K.K. Likharev. JETP 88, 692 (1985).

[11] K.K. Likharev, A.B. Zorin. J. low Temp. Phys. 59 3/4, 347 (1985). 
[12] A.M. Hriscu, Y.V. Nazarov. Phys. Rev. B 83, 174511 (2011).

[13] M. Zgirski, K.-P. Riikonen, V. Tuboltsev, P. Jalkanen, T.T. Hongisto, K.Yu. Arutyunov. Nanotechnology 19, 055301 (2008).

[14] J.S. Lehtinen, T. Sajavaara, K.Yu. Arutyunov, M.Yu. Presnjakov, A. Vasiliev. Phys. Rev. B 85, 094508 (2012).

[15] A.D. Zaikin, D.S. Golubev, A. van Otterlo, G.T. Zimányi. Phys. Rev. Lett. 78, 1552 (1997).

[16] D.S. Golubev, A.D. Zaikin. Phys. Rev. B 64, 014504 (2001).

[17] J.S. Lehtinen, K. Zakharov, K. Arutyunov. Phys. Rev. Lett. 109, 187001 (2012).

[18] L. Kuzmin, Y. Pashkin, T. Claeson. Supercond. Sci. Tech. 7, 324 (1994).

[19] M. Watanabe, D.B. Haviland. Phys. Rev. Lett. 86, 5120 (2001).

Редактор Т.Н. Василевская 